\title{
INFLUENCE OF MODEL ERRORS IN OPTIMAL SENSOR PLACEMENT
}

Loris Vincenzi*, Laura Simonini

University of Modena and Reggio Emilia, Department of Engineering "Enzo Ferrari”

Via Pietro Vivarelli 10, 41125, Modena, Italy.

* corresponding author: loris.vincenzi@unimore.it

\begin{abstract}
The paper investigates the role of model errors and parametric uncertainties in optimal or near optimal sensor placements for structural health monitoring (SHM) and modal testing. The near optimal set of measurement locations is obtained by the Information Entropy theory; the results of placement process considerably depend on the so-called covariance matrix of prediction error as well as on the definition of the correlation function. A constant and an exponential correlation function depending on the distance between sensors are firstly assumed; then a proposal depending on both distance and modal vectors is presented. With reference to a simple case-study, the effect of model uncertainties on results is described and the reliability and the robustness of the proposed correlation function in the case of model errors are tested with reference to $2 \mathrm{D}$ and $3 \mathrm{D}$ benchmark case studies. A measure of the quality of the obtained sensor configuration is considered through the use of independent assessment criteria. In conclusion, the results obtained by applying the proposed procedure on a real 5-spans steel footbridge are described. The proposed method also allows to better estimate higher modes when the number of sensors is greater than the number of modes of interest. In addition, the results show a smaller variation in the sensor position when uncertainties occur.
\end{abstract}

Keywords: Optimal sensor placement, Information Entropy, Model error, Model uncertainties

\section{Introduction}

Modern technologies for structural safety, system identification, and damage detection require control systems to monitor the structural behaviour during the whole operating life. These technologies are based on the development of efficient numerical techniques for structural identification and on the adoption of increasingly reliable sensors that conjugate contained costs with performances suitable for the monitoring purposes. However, the quality of the obtained information significantly depends on the number of sensors and on their positions. Usually, several alternative positions can be selected, although economic constraints and spatial restrictions tend to limit the sensor set-up. Thus, it is necessary to optimize the position of a limited number of sensors in order to obtain the maximum amount of information from the measurements and to assure a reliable evaluation of the parameters of interest.

As a matter of facts, sensor placement is a priori problem where only analytical data are available; they are obtained, for instance, by means of a Finite Element Model. Therefore, in a general sensor placement procedure, the estimate of the optimal position is sensitive to errors and uncertainties of the numerical model. They can alter the optimal locations of sensors and limit the efficiency of the monitoring systems. These uncertainties are mainly due to limitations of the adopted numerical models to represent the behaviour of the real structure (model errors) and the presence of uncertainties 
in measures whether experimental data are used (measurement errors). Moreover, model errors can be further classified in two groups: parametric uncertainties and model form uncertainties. The parametric uncertainties are associated with the discrepancies between some parameter values of the actual physical system and with the input parameters in the numerical model. Model form uncertainties are associated with the inaccuracy in modeling of the physical system [1].

In this work, the role of parametric and model uncertainties in sensor placements is investigated. The Information Entropy [2] method is applied. According to [3], the covariance matrix of the prediction error is defined by a term counting the measurement error which is assumed as independent from the sensor position and by a term that considers the model error. If the goal of the monitoring process is the modal identification, the Information Entropy only depends on the modal matrix and on the prediction error of the signal acquired by two sensors in hypothetical locations. Thus, results of optimization and placement process considerably depend on the covariance matrix of prediction error and on the definition of the signal correlation. The role of the covariance matrix and the correlation function in optimal sensor placement is thus investigated considering at first a simple case-study without uncertainties. Some approaches in the definition of covariance matrix are compared; firstly it is assumed a constant correlation function and then an exponential correlation function depending on the distance between sensors [3]. A new proposal that depends on both the distance and modal vectors is then presented. The reliability and the robustness of the proposed correlation function in the case of model error are also tested by forcing an alteration (distortion) in a constraint condition. A measure of the quality of the obtained sensor configuration is evaluated through the use of independent assessment criteria. Spatially correlated prediction errors are considered with reference to an asymmetric-plan multi-storey building. Finally, the real case study of a five spans steel footbridge is presented.

The investigation of the model errors (model form uncertainties and parametric uncertainties) in optimal sensor placement and a new proposal for the correlation function are the main contributions of this paper; it will show that the proposed correlation function easily allows to take into account the spatial correlation in 3D structures and it could reduce the variability of results in the case of model uncertainties.

\section{Related works}

In the last decades, several approaches have been proposed and developed to find a sensor configuration that allows to optimize the number and the position of sensors. An overview of the optimization criteria for optimal placement of sensors and actuators on a smart structure is presented in [4]. One set of criteria was derived from the modal assurance criterion (MAC) originally introduced by [5]. With the aim of leading in uncorrelated mode shapes, [6] proposed to minimize the off diagonal terms of the MAC matrix. The MAC or the mass-weighted MAC were recommended as a validation criterion by several authors [7]. In the field of optimal sensor placement, [8] proposed to minimize both the average of all the off-diagonal elements and the highest value in all of them.

In [9] a priori analysis was performed; the proposed approach to optimal sensor placement is based on the difference among modal information acquired with or without the contribution of preselected sensors. A lack of information about the $i$-th sensor implies that the mode shape component is estimated by a linear interpolation. 
The Effective Independence (EFI) method [10-12] was developed for spatial structures on orbit. It determines the position of each candidate sensor maximizing the determinant of the Fisher Information Matrix (FIM) defined as the product of the mode shape matrix and its transpose. The aim of the EFI method is to select measurement positions that make the mode shapes a readings of interest as linearly independent as possible. The EFI method is extensively discussed in papers [11-14]. The Fisher Information Matrix can also be weighted by the use of the mass matrix carried out by a finite element model [15]. In the EFI method, the number of sensors is iteratively reduced from an originally large candidate set to the desired number, by removing those sensors which do not contribute significantly to the independent information of the mode partition [16]. In the end, the remaining sensors are judged as the optimal sensor set. In [13] it is shown that maximizing the FIM determinant means to maximize the mode shape signal strength and spatial independence.

The determinant of the Fisher Information matrix is often directly considered as the objective function that have to be minimized; other authors suggests to maximize the smallest eigenvalue of the FIM (see for instance [17]), or to minimize the trace of inverse of the FIM [18], or to maximize the norm of the FIM $[19,20]$. Different variants based on the EFI method were also proposed [21]: for instance, the EFI-DPR (Driving Point Residue) was developed to identify the damage in mechanical elements $[10,11,13,22]$ and the modes are weighted by corresponding driving-point residues.

Several other sensor placement methods are based on energy measures of a structure. These methods usually select locations with high amplitude responses to increase the signal to noise ratio. The Kinetic Energy Method (KEM) [23] maximizes the kinetic energy measure of the structure; however, it is shown that it should be brought back to the maximization of the Fisher information matrix determinant [10]. The idea of maximize the signal to noise ratio of reference sensors is also presented in the method proposed by [24]. Other energy-driven methods are the Eigenvalue Vector Product (EVP) [25] and the Non-Optimal Driving Point (NODP) [26].

Papadimitriou [2] introduces the Information Entropy (IE) as a performance measure of the sensor configuration. The method generalizes several other sensor placement procedures and it demonstrates its efficiency and robustness. Based on the Bayesian approach, the Information Entropy provides for a scalar measure of the uncertainty in the estimate of some model parameters and it depends on the covariance matrix of prediction error. The optimal sensor configuration is the one that minimizes uncertainties, that is the IE.

In recent years, some researches have been focused on incorporating the uncertainties associated with real structures into the numerical simulation for reliable predictions. Despite this, only few studies investigate the optimal placement of sensors with model errors [27]. In [1] the influence of parametric uncertainties on the optimal sensor placement methodologies for the modal analysis of a truss bridge is investigated. Four classical sensor location methodologies are employed; two of these are based on the Fisher Information matrix and the other two are based on energy optimization [28].

In this work, the role of parametric and model uncertainties in sensor placements is investigated by means of the Information Entropy [2] method. A brief overview of the method in the field of sensor placement is reported in the next section. 


\section{The Information Entropy}

For a $n$ Degree Of Freedom (DOF) linear vibratory system, the measured response $\mathbf{y}(t)$, depending on the time $t$, is related to the model response $\mathbf{x}(t ; \boldsymbol{\theta})$ by the observation equation

$\mathbf{y}(t)=\mathbf{L} \mathbf{x}(t ; \boldsymbol{\theta})+\mathbf{e}(t ; \boldsymbol{\theta})$

where the vector $\mathbf{x}(t ; \boldsymbol{\theta})$ is the response displacement and $\mathbf{e}(t ; \boldsymbol{\theta})$ is the prediction error due to the model error and the measurement error; $\mathbf{L}$ is the observation matrix, composed of zeros and ones, that specifies which DOFs of the system are measured. Moreover, $\boldsymbol{\theta}$ are parameters characterizing the dynamic structural behaviour. According to the Bayesian theory, the Probability Density Function $p\left(\boldsymbol{\theta} \mid \boldsymbol{\Sigma}_{t}, D\right)$ of parameters $\boldsymbol{\theta}$, obtained from the data $D$ measured on the system, is given by [2]:

$p\left(\boldsymbol{\theta} \mid \boldsymbol{\Sigma}_{t}, D\right)=\frac{c}{(\sqrt{2 \pi})^{N} \sqrt{\operatorname{det}\left(\boldsymbol{\Sigma}_{t}\right)}} \exp \left(-\frac{N N_{0}}{2} \mathbf{J}\left(\boldsymbol{\theta} \mid \boldsymbol{\Sigma}_{t}, D\right)\right) \pi(\boldsymbol{\theta})$

where $\pi(\boldsymbol{\theta})$ is a priori distribution of the parameter $\boldsymbol{\theta}, c$ is a normalizing constant chosen such that the Probability Density Function (PDF) in Eq. 2 integrates to one and $\boldsymbol{\Sigma}_{t}$ is the covariance matrix of the prediction error $\mathbf{e}(t ; \boldsymbol{\theta}) . \mathbf{J}\left(\boldsymbol{\theta} \mid \boldsymbol{\Sigma}_{t}, D\right)$ represents the distance between the measured data and the model response time histories:

$\mathbf{J}\left(\boldsymbol{\theta} \mid \boldsymbol{\Sigma}_{t}, D\right)=\frac{1}{N N_{0}} \sum_{k=1}^{N}\left[\mathbf{y}_{k}-\mathbf{L} \mathbf{x}_{k}(\boldsymbol{\theta})\right]^{T} \boldsymbol{\Sigma}_{t}^{-1}\left[\mathbf{y}_{k}-\mathbf{L} \mathbf{x}_{k}(\boldsymbol{\theta})\right]$

$N_{0}$ is the number of the sensors and $N$ is the time interval length. Since the PDF is a measure of uncertainties in parameter values, the Information Entropy [29]

$\mathbf{h}\left(\mathbf{L} \mid \boldsymbol{\Sigma}_{t}, D\right)=E_{\theta}\left[-\ln \left(p\left(\boldsymbol{\theta}, \boldsymbol{\Sigma}_{t} \mid D\right)\right)\right]=-\int \ln \left(p\left(\boldsymbol{\theta}, \boldsymbol{\Sigma}_{t} \mid D\right)\right) p\left(\boldsymbol{\theta}, \boldsymbol{\Sigma}_{t} \mid D\right) d \boldsymbol{\theta}$

provides a unique scalar measure of the uncertainty in the evaluation of structural parameters. $\mathrm{E}_{\theta}\left[{ }^{\cdot}\right]$ denotes the expected value of $p\left(\boldsymbol{\theta} \mid \boldsymbol{\Sigma}_{t}, D\right)$ towards $\boldsymbol{\theta}$. An asymptotic approximation of $\mathbf{h}\left(\mathbf{L} \mid \boldsymbol{\Sigma}_{t}, D\right)$ is available for a large number of acquired data, i.e. for $N N_{0} \rightarrow \infty[2,22]$

$\mathbf{h}\left(\mathbf{L} \mid \boldsymbol{\Sigma}_{t}, D\right) \approx \mathbf{h}\left(\mathbf{L}, \boldsymbol{\Sigma}_{t}, \boldsymbol{\theta}\right)=1 / 2 N_{0} \ln (2 \pi)-\ln \left[\operatorname{det}\left(\mathbf{Q}\left(\mathbf{L}, \boldsymbol{\Sigma}_{t}, \boldsymbol{\theta}\right)\right)\right] / 2$

where $\mathbf{Q}\left(\mathbf{L}, \boldsymbol{\Sigma}_{t}, \boldsymbol{\theta}\right)$ is the Fisher Information Matrix (FIM). Its asymptotic approximation is [2]

$\mathbf{Q}\left(\mathbf{L}, \boldsymbol{\Sigma}_{t}, \boldsymbol{\theta}\right) \approx \sum_{k=1}^{N}\left[\mathbf{L} \nabla_{\theta} \mathbf{x}_{k}(\boldsymbol{\theta})\right]^{T}\left[\mathbf{L} \boldsymbol{\Sigma}_{t} \mathbf{L}^{T}\right]^{-1}\left[\mathbf{L} \nabla_{\theta} \mathbf{x}_{k}(\boldsymbol{\theta})\right]$

in which $\nabla_{\theta}$ is the gradient operator. The optimal sensor configuration is selected as the one that minimizes the Information Entropy since it gives a direct measure of uncertainty in the model parameter estimate. Eq.5 easily shows that to minimize the Information Entropy corresponds to maximize the determinant of the Fisher Information Matrix

$\mathbf{L}_{\text {best }}=\underset{\mathbf{L}}{\operatorname{argmin}}\left[\mathbf{h}\left(\mathbf{L}, \boldsymbol{\Sigma}_{t}, \boldsymbol{\theta}\right)\right]=\underset{\mathbf{L}}{\operatorname{argmax}}\left[\operatorname{det}\left(\mathbf{Q}\left(\mathbf{L}, \boldsymbol{\Sigma}_{t}, \boldsymbol{\theta}\right)\right)\right]$ 
If the aim of the structural monitoring is the modal identification, the best sensor position provides for the best amount of information on the modal coordinate vector $\xi$ [3]. Thus, the model parameter vector corresponds to the modal coordinate vector, that is $\boldsymbol{\theta}=\boldsymbol{\xi}$. Using the coordinate transformation, the structural response displacement vector $\mathbf{x}$ is written as the product of the modal coordinate vector and the mode shape matrix $\boldsymbol{\Phi}=\left\lfloor\boldsymbol{\varphi}_{1}, \ldots, \boldsymbol{\varphi}_{k}, \ldots \boldsymbol{\varphi}_{N_{M}}\right\rfloor$ :

$\mathbf{x}=\boldsymbol{\Phi} \xi=\boldsymbol{\Phi} \boldsymbol{\theta}$

Considering that $\nabla_{\theta} \mathbf{x}=\boldsymbol{\Phi}$ and substituting it into Eq.6, the Fisher Information matrix takes the form

$\mathbf{Q}\left(\mathbf{L}, \boldsymbol{\Sigma}_{t}, \boldsymbol{\theta}\right)=[\mathbf{L} \boldsymbol{\Phi}]^{T}\left[\mathbf{L} \boldsymbol{\Sigma}_{t} \mathbf{L}^{T}\right]^{-1}[\mathbf{L} \boldsymbol{\Phi}]$

Therefore, the Fisher Information Matrix is independent from the modal coordinates $\xi$ and it is connected to the sensor positions both by the observation matrix $\mathbf{L}$ and by the mode shape matrix $\boldsymbol{\Phi}$

\subsection{The covariance matrix of the prediction error}

The definition of the covariance matrix $\boldsymbol{\Sigma}_{t}$ of the prediction error $\mathbf{e}(t ; \boldsymbol{\theta})$ is a crucial issue for the optimal sensor position, especially in presence of model uncertainties. In general, the prediction error depends on a term counting the measurement error, usually unrelated to the sensor position, and on a term that considers the model error [3]. Assuming the independence between these two errors, the covariance $\boldsymbol{\Sigma}_{t}$ is given by

$$
\Sigma_{t}=\bar{\Sigma}+\boldsymbol{\Sigma}
$$

where $\overline{\boldsymbol{\Sigma}}$ and $\boldsymbol{\Sigma}$ are respectively the measurement error and model error covariance matrices.

The measurement error matrix $\bar{\Sigma}$ is usually independent from the sensor position and, in common applications, it is assumed diagonal. Off diagonal terms could be considered only for multi-axial digital sensors where measures are collected by sensors physically allocated on the same chip (see for instance [30]). Generally the model error covariance matrix $\boldsymbol{\Sigma}$ depends on the distance between sensors; therefore, the prediction error of the structural response has a correlation among the measures acquired in neighbouring points. In [3] an exponential correlation function for DOFs $i$ and $j$ is assumed:

$\Sigma_{i j}=\sqrt{\sum_{i i} \Sigma_{j j}} \exp \left(-\delta_{i j} / \lambda\right)$

where $\delta_{i j}$ is the spatial distance between the DOFs $i$ and $j$, and $\lambda$, called correlation length, is a measure of the spatial correlation in signals. The auto-correlation terms $\Sigma_{i i}, \Sigma_{j j}$ are assumed equal to one. The cross-correlation term $\Sigma_{i j}$ spans from zero to one; it is equal to one in the case of perfect spatial correlation and tends to zero when the distance between sensors increases. 
In [3] the role of correlation length is investigated, considering different constant values of $\lambda$. In this paper, a new correlation function is defined. For two given position $i$ and $j$, elements of the model error covariance matrix are assumed as

$$
\Sigma_{i j}=\frac{\boldsymbol{\psi}_{i} \cdot \boldsymbol{\psi}_{j}^{T}}{N_{M}} \exp \left(-\delta_{i j} / \bar{\delta}\right)
$$

where $\bar{\delta}$ represents an average distance among sensors and it is defined as the ratio between the greatest distance of all DOFs and the number of sensors; $N_{M}$ is the number of modes and $\boldsymbol{\psi}_{i}=\left[\psi_{i, 1}\right.$ $\left.\psi_{i, 2} \quad \cdots \quad \psi_{i, k}\right], \boldsymbol{\Psi}_{j}=\left[\begin{array}{lllll}\psi_{j, 1} & \psi_{j, 2} & \cdots & \psi_{j, k}\end{array}\right]$ are vectors whose terms are:

$\psi_{i, k}=\frac{\left|\varphi_{i k}\right|}{\max \left(\left|\varphi_{i k}\right| ; \mid \varphi_{j k}\right)} ; \quad \psi_{j, k}=\frac{\left|\varphi_{j k}\right|}{\max \left(\left|\varphi_{i k}\right| ;\left|\varphi_{j k}\right|\right)}$.

The distance between two sensors is thus combined with information given by mode shapes, computed in the $i$-th and $j$-th position ( $\varphi_{i k}$ and $\varphi_{j k}$ respectively) for each contributing mode $k$. Due to its definition, $\psi_{i, k}$ and $\psi_{j, k}$ vary in the range ]0,1]; in the case of the indeterminate solution $\psi_{i, k}=\frac{0}{0}$ the value of $\psi_{i, k}$ is imposed equal to one because both mode shapes components given by sensors $i$ and $j$ are equal to zero and, then, information are fully correlated. The product $\boldsymbol{\psi}_{i} \cdot \boldsymbol{\psi}_{j}^{T}$ varies between 0 and the number of modes, giving a weight for the distance-dependent factor $\exp \left(-\delta_{i j} / \bar{\delta}\right)$. The auto-correlation terms $\Sigma_{i i}$ are still assumed equal to one.

The value of the element $\Sigma_{i j}$ assumes the maximum value equal to one when the information is totally correlated and it decreases with the distance between sensors. In the general case, high correlation is obtained when sensors are close to each other.

Furthermore, the product $\boldsymbol{\psi}_{i} \cdot \boldsymbol{\psi}_{j}^{T}$ easily allows to take into account the correlation in 3D structures. Consider for instance for a multi-storey building having rigid floor diaphragms and planwise distribution of mass and stiffness symmetric in two directions. Due to the symmetry, the vibration modes are uncoupled in the horizontal directions. For two sensors oriented in these two orthogonal directions, the product $\boldsymbol{\psi}_{i} \cdot \boldsymbol{\psi}_{j}^{T}$ is zero and, then, $\Sigma_{i j}$ is equal to 0 even if the distance between sensors is small. On the contrary, for coupled lateral-torsional modes in asymmetric-plan frames, the product $\boldsymbol{\psi}_{i} \cdot \boldsymbol{\psi}_{j}^{T}$ gives a weight for the distance-dependent factor $\exp \left(-\delta_{i j} / \bar{\delta}\right)$ taking into account the spatial correlation between sensors oriented in different direction.

\section{The optimization process}

Several algorithms are available to find the configuration that minimizes the Information Entropy (or maximizes the determinant of the Fisher Information Matrix). The most common algorithms are Genetic (GA) and Evolutionary algorithms (EA) [31, 32], Simulated Annealing algorithm (SA) [23] 
and Particle Swarm Optimization algorithm (PSO). They imitate the natural evolution by assigning a fitness value to each candidate and applying the principle of gene survival or other natural development to find the optimal solution.

The main issue of the optimization process is to find an efficient searching solution in order to generate acceptable results in reasonable time. The Sequential Sensor Positioning procedures (SSP) are much more computationally efficient with respect to exhaustive search methods and to natureinspired algorithms. The sensor configuration computed by the SSP algorithm cannot be guaranteed to be the optimal one; however numerical applications show that the results coincide with, or are very close to the exact solution [2].

In this work the Forward Sequential Sensor Placement (FSSP) procedure is applied. The FSSP procedure is a sub-case of SSP algorithm and, differently from the Backward Sequential Sensor Placement, it puts sensors on the structure one at a time, evaluating, for each subsequent sensor, which position maximizes the objective function. The computational effort involved in the FSSP procedure is much smaller than the ones involved for a direct search method, especially when a large discard between the DOF number and the number of sensors is considered [3].

The optimization procedure computes at first the covariance matrices (only once at the beginning of the process), according to equations reported in the subsection 3.1. Then, the FIM $\mathbf{Q}(\mathbf{L})$ is evaluated with reference to only the first sensor. The matrix $\mathbf{L}$ has then only one row and it is full of zeros except to the DOF where the sensor is placed. The position that maximizes the determinant of the FIM is selected as the best one. Further sensors are applied with the same criterion; the dimension of $\mathrm{L}$ is increased and the optimal sensor position found in the previous step is maintained.

When the optimization algorithm is applied to minimize the Information Entropy (Eq. 7), some numerical problems could occur. In particular, the optimization process exhibits an indeterminate solution in the case of a limited number of sensors [33]: the determinant of the FIM (Eq.9) is zero for all sensor configurations if the number of sensor $N_{0}$ is smaller than the contributing mode number $N_{M}$. To avoid the aforementioned indetermination, a proper numerical procedure has to be adopted. The Fisher Information matrix is a real symmetric matrix and, applying the diagonalization process, it is possible to find a diagonal matrix similar to $\mathbf{Q}\left(\mathbf{L}, \boldsymbol{\Sigma}_{t}, \boldsymbol{\theta}\right)$ with its eigenvalues on the diagonal; the determinant is then simply computed by the product of the diagonal elements (i.e. the eigenvalues of Q). Papadimitriou and Lombaert [3] proposed to solve the problem maximizing only the non-zero eigenvalue product of the FIM, instead of maximizing the product of all eigenvalues. However, for numerical approximations in the diagonalization procedure and in the evaluation of the term $\left(\mathbf{L} \boldsymbol{\Sigma}_{t} \mathbf{L}^{T}\right)^{-1}$, some near-zero values could appear. They can be taken into consideration or neglected depending on the imposed threshold value, giving wide fluctuations to results.

In this paper, a more robust procedure is applied. The FIM and, thus, the term $\left(\mathbf{L} \boldsymbol{\Sigma}_{t} \mathbf{L}^{T}\right)^{-1}$ is a positive definite matrix. A Cholesky factorization of the term $\left(\mathbf{L} \boldsymbol{\Sigma}_{t} \mathbf{L}^{T}\right)^{-1}$ is firstly performed $\left(\mathbf{L} \boldsymbol{\Sigma}_{t} \mathbf{L}^{T}\right)^{-1}=\mathbf{C} \mathbf{C}^{T}$

so obtaining 
$\mathbf{Q}\left(\mathbf{L}, \boldsymbol{\Sigma}_{t}, \boldsymbol{\theta}\right)=[\mathbf{L} \boldsymbol{\Phi}]^{T} \mathbf{C} \mathbf{C}^{T}[\mathbf{L} \boldsymbol{\Phi}]=\mathbf{A}^{T} \mathbf{A}$

where $\mathbf{A}=\mathbf{C}^{T}[\mathbf{L} \boldsymbol{\Phi}]$ is a $N_{0} \times N_{M}$ matrix, $N_{0}$ is the number of sensors and $N_{M}$ is the number of modes. Then, the Singular Value Decomposition of $\mathbf{A}$ is computed. Denoting with $\sigma_{\mathrm{a}}(\mathbf{A})$ the square of the $z$-th singular value of $\mathbf{A}$, the determinant of $\mathbf{Q}$ is estimated by the product among $\sigma_{\mathrm{a}}(\mathbf{A})$; the best configuration is evaluated according to

$$
\left.\mathbf{L}_{\text {best }}=\underset{\mathbf{L}}{\operatorname{argmax}}\left[\operatorname{det}\left(\mathbf{Q}\left(\mathbf{L}, \boldsymbol{\Sigma}_{t}, \boldsymbol{\theta}\right)\right)\right]=\underset{\mathbf{L}}{\operatorname{argmax}} \mid \prod_{\mathrm{a}} \sigma_{\mathrm{a}}(\mathbf{A})\right]
$$

The proposed procedure has lower computational effort and gives more stable results instead of considering the non-zero eigenvalues.

\section{Numerical examples}

In the next subsections two simple numerical case studies are presented to investigate the role played by the covariance matrix of the prediction error and how uncertainties in the structural model could modify results of the sensor placement.

The first case study refers to a simply supported beam; first, the proposal described in Eq. 12 is compared with the one proposed in [3] and reported in Eq. (11). Then, the reliability and the robustness of the proposed correlation function in the case of model form uncertainty are tested forcing an alteration (distortion) in a constraint condition. A measure of the quality of the obtained sensor configuration is considered through the use of independent assessment criteria.

In the second case, the proposed correlation function is applied to an eight-storey asymmetric-plan building; it will be shown that the proposed correlation function easily takes into account the spatial correlation in 3D structures. Then, the sensor placement procedure is performed starting from a set

of pseudo-experimental data, which simulate the statistical scattering of some physical parameters that are due to uncertainties in the model definition.

\subsection{Example 1: simply supported beam}

The role of the covariance matrix and the correlation function in optimal sensor placement is firstly investigated referring to a simply supported beam with length $L=1$. Procedures described in Section 3 and 4 are implemented and the Information Entropy approach is applied with the FSSP procedure.

Numerical test has been firstly performed adopting exact values of mode shape vectors as input data (no measurement or model errors are considered). A total of $N_{M}=4$ contributing modes is selected and 500 possible positions equally arranged on the beam length are considered. Mode shapes are normalized so that the largest component is equal to one.

The results obtained by different formulation are compared. The first formulation assumes $\boldsymbol{\Sigma}$ as an identity matrix and, thus, neglects the correlation in the covariance matrix. In the following, it is denoted as case A. Case B indicates the case where only the distance-dependent correlation in the covariance matrix is considered. It is computed according to Eq.(11). Finally, the correlation proposed in this paper (Eq.12) is denoted as case C. 
The results concerning the position of the first 6 sensors for the simply supported beam are shown in Figure 1. All formulations (cases A, B and C) place the first 4 sensors almost in the same position. Only the placement order is changed for the case B (Figure 1b). Greater differences are instead found in the position of subsequent sensors. Neglecting the correlation between sensors, the case A tends to place the $5^{\text {th }}, 6^{\text {th }}$ and $7^{\text {th }}$ sensors very close to the previous positions. Also next sensors (not given in figure) are placed with the same criterion, so obtaining a crowded concentration of sensors in the neighbouring of only 4 positions. This result is expected: in an error-free case study the optimization problem is fully determined when the number of sensors is equal to the number of modes and thus the first 4 sensors can optimally describe the selected 4 mode shapes. Guided by the aim to well space the sensors, case $\mathrm{B}$ assigns the $5^{\text {th }}$ and $6^{\text {th }}$ sensor close to the beam ends. No useful information about mode shapes is substantially added even if sensors are well spaced. Case $\mathrm{C}$ defines new positions that are closed to the maximum value of the $2^{\text {nd }}$ and $3^{\text {rd }}$ mode shapes and the proposed formulation appears to improve the results obtained for case $\mathrm{A}$ and $\mathrm{B}$, because it spaces better the sensors and avoids useless nodal positions.

A measure of the quality of the sensor configurations is necessary in order to numerically compare the obtained results. Three independent assessment criteria are selected to evaluate the suitability of selected sensor position: the determinant of Fisher Information Matrix, a criterion based on the Modal Assurance Criterion (MAC) and the so called Condition Number (CN). These criteria are independent of each other and they are considered very reliable [1]. These criteria are briefly described below. It can be easily remarked that these assessment criteria do not give absolute judgments to the quality of the obtained configurations but they are used only to compare different lay-out. Further information can be found in [7].

The determinant of the Fisher Information Matrix is reported in Eq. (9) in the extended formulation. To compare the results, the expression without the covariance matrix of the prediction error is used:

$$
\operatorname{det}(\mathrm{FIM})=\operatorname{det}\left(\hat{\boldsymbol{\Phi}}^{T} \hat{\boldsymbol{\Phi}}\right)=[\mathbf{L} \boldsymbol{\Phi}]^{T}[\mathbf{L} \boldsymbol{\Phi}]
$$

$\hat{\mathbf{\Phi}}$ is the mode shape matrix evaluated at only the measured DOFs. A high value of this parameter is preferred as it indicates that the FIM retains as much information as possible [13].

The Modal Assurance Criterion (MAC, [34]) is traditionally used to correlate numerical mode shapes to those obtained by experimental tests. In the field of optimal sensor placement, the mode shape data are really useful when modes can be distinguished one from each other; a good sensor configuration has then off diagonal terms as small as possible. Then, the root mean square (RMS) of the off-diagonal MAC terms is used as an assessment criterion:

$$
\mathrm{RMS}=\sqrt{\frac{1}{N_{M}\left(N_{M}-1\right)} \sum_{h=1}^{N_{M}} \sum_{\substack{k=1 \\ k \neq h}}^{N_{M}}\left(\mathrm{MAC}_{h k}\right)^{2}}
$$

where the MAC is defined as

$$
\mathrm{MAC}_{h k}=\frac{\left(\hat{\boldsymbol{\varphi}}_{h}{ }^{T} \hat{\boldsymbol{\varphi}}_{k}\right)^{2}}{\left(\hat{\boldsymbol{\varphi}}_{h}{ }^{T} \hat{\boldsymbol{\varphi}}_{h}\right)\left(\hat{\boldsymbol{\varphi}}_{k}{ }^{T} \hat{\boldsymbol{\varphi}}_{k}\right)}
$$


$\hat{\boldsymbol{\varphi}}_{h}$ and $\hat{\boldsymbol{\varphi}}_{k}$ are the $h$-th and the $k$-th modal vectors computed only in the selected DOFs. Small values of the RMS indicate good correlations.

The Condition Number (CN) is calculated using the Singular Value Decomposition (SVD) of the eigenvector matrix. The $\mathrm{CN}$ is defined as the ratio between the largest and the smallest non-zero singular value of mode shape matrix kept by measured DOFs. Large condition number indicates a poor choice of sensor configuration.

Finally, a summary index IR is introduced:

$I R=\frac{1}{3}\left[\frac{R M S_{\min }}{R M S_{z}}+\frac{\mathrm{CN}_{\min }}{\mathrm{CN}_{z}}+\frac{(\operatorname{det}(\mathrm{FIM}))_{z}}{(\operatorname{det}(\mathrm{FIM}))_{\max }}\right]$

where $\mathrm{RSM}_{z}, \mathrm{CN}_{z}$ and $\operatorname{det}(\mathrm{FIM})_{z}$ are calculated for the $\mathrm{z}$-th case $(\mathrm{A}, \mathrm{B}$ or $\mathrm{C})$ and $\mathrm{RSM}_{\min }, \mathrm{CN}_{\min }$, $\operatorname{det}(\mathrm{FIM})_{\max }$ are the best indices among all cases. Value of IR equal to one means that the method provides the best indices for all criteria.

The assessment criteria have been applied to optimal configurations for cases A, B and C; the results are reported in Table 1. Values of assessment criteria are given in consideration of the first 6 sensors or 7 sensors. It can be noted that no formulation gives a sensor placement that satisfies better all criteria. The Case A obviously satisfies the FIM criterion; the case B has lower values of RMS and $\mathrm{CN}$ when the first 6 sensors are selected while the case $\mathrm{C}$ gives better results if 7 sensors are considered. However, case $\mathrm{C}$ has high value of the IR index both if 6 or 7 sensors are selected.

The sensor placement procedure needs to assign a priori the number of modes. The placement process described and used in this paper assumes that the number of sensors is equal to the number of target modes that should be identified at least [29]. With a number of sensors exactly equal to the number of modes, the problem is fully determined; indeed, results show that for all cases the first four modes are identified with the first 4 sensors with the same accuracy and almost coincident positions are obtained for all cases. When the number of sensors is greater than the number of modes, in our opinion, the optimal sensor configuration is the one that allows to identify as better as possible the greatest number of modes. With this aim, the assessment criteria are recalculated supposing that 5 mode shapes are actually available even if the optimal sensor procedure is carried out taken into account only the firsts 4 mode shapes. Results are given in Table 2. The proposed correlation function (case C) better satisfies all assessment criteria with a large discard with respect to the other cases, both for the case of 6 and 7 sensors. Similar results can be obtained for a higher number of modes.

\subsubsection{Example 1: simply supported beam with model uncertainty}

In this subsection, a model form error is introduced and the results of the three formulation described in the subsection 5.1 are compared. We suppose that the beam has a rotational spring with stiffness $K$ at one end; however, with a lack of information on the actual constraint condition, the sensor placement is performed using a simply supported beam model. Therefore, the results of the placement procedure are those already described in Figure 1. For a beam with a rotational spring at the right end, the free-vibration equation of motion is solved with the general solution in the form [24]:

$v=A_{1} \cos (\alpha x)+A_{2} \sin (\alpha x)+A_{3} \cosh (\alpha x)+A_{4} \sinh (\alpha x)$ 


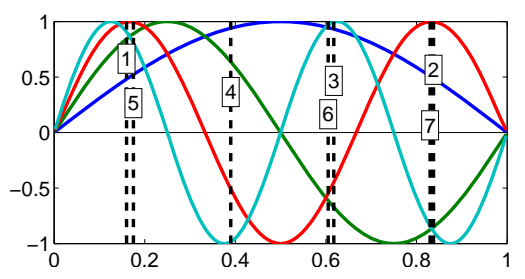

(a) Case $\mathrm{A}$

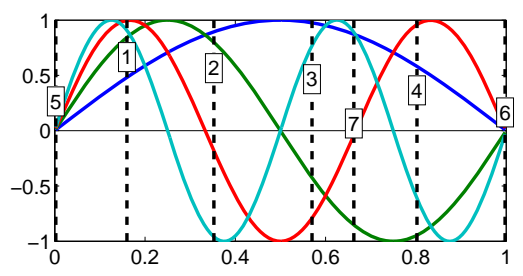

(b) Case B

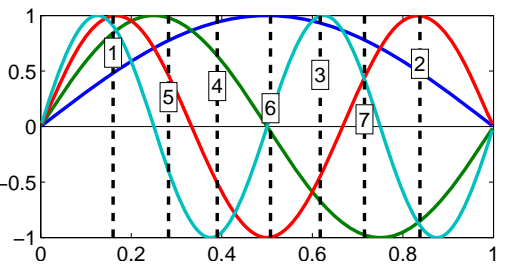

(c) Case $\mathrm{C}$

Figure 1 Position of sensors in the simply supported beam

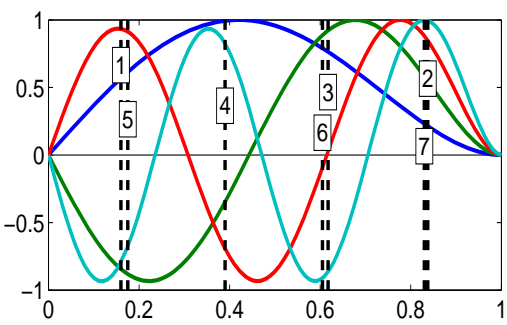

(a) Case A

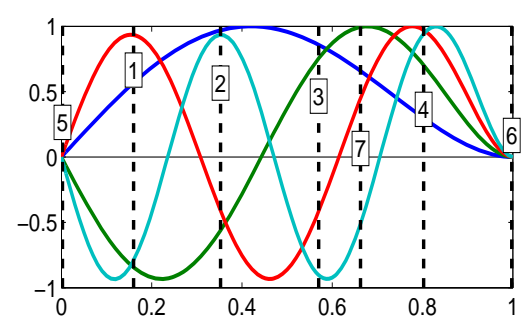

(b) Case B

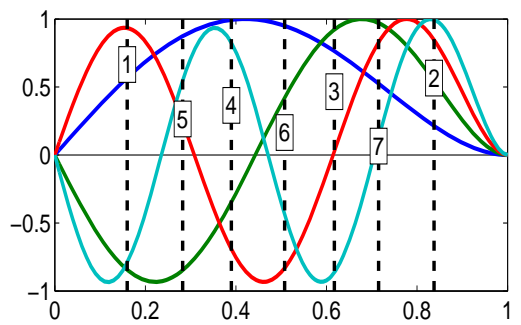

(c) Case $\mathrm{C}$

Figure 2 Mode shapes of the beam with a spring at the right end. Position of sensors is defined according to a simply supported scheme.

Table 1 Example no. 1. Assessment criteria values -4 modes, 6 or 7 sensors

\begin{tabular}{|c|c|c|c|c|c|c|c|c|c|}
\hline \multicolumn{5}{|c|}{6 sensors } & \multicolumn{5}{|c|}{7 sensors } \\
\hline & $\operatorname{det}(F I M)$ & RMS & $\mathbf{C N}$ & IR & & $\operatorname{det}(F I M)$ & RMS & $\mathbf{C N}$ & IR \\
\hline $\mathbf{A}$ & 179.06 & 0.06 & 1.61 & 0.596 & $\mathbf{A}$ & 358.67 & 0.03 & 1.65 & 0.681 \\
\hline B & 38.54 & 0.004 & 1.16 & 0.738 & B & 78.64 & 0.03 & 1.40 & 0.463 \\
\hline C & 154.77 & 0.02 & 1.36 & 0.639 & $\mathbf{C}$ & 275.15 & 0.01 & 1.17 & 0.922 \\
\hline
\end{tabular}

Table 2 Example no. 1. Assessment criteria values -5 modes, 6 or 7 sensors

\begin{tabular}{|c|c|c|c|c|c|c|c|c|c|}
\hline \multicolumn{5}{|c|}{6 sensors } & \multicolumn{5}{|c|}{7 sensors } \\
\hline & $\operatorname{det}($ FIM) & RMS & $\mathrm{CN}$ & IR & & $\operatorname{det}(F I M)$ & RMS & $\mathbf{C N}$ & IR \\
\hline $\mathbf{A}$ & 9.12 & 0.30 & 12.27 & 0.087 & $\mathbf{A}$ & 19.89 & 0.30 & 12.06 & 0.053 \\
\hline B & 0.11 & 0.26 & 42.48 & 0.052 & B & 94.89 & 0.05 & 2.21 & 0.288 \\
\hline $\mathrm{C}$ & 353.94 & 0.03 & 1.65 & 1.000 & $\mathbf{C}$ & 964.54 & 0.01 & 1.25 & 1.000 \\
\hline
\end{tabular}

where $\alpha^{4}=m \omega^{2} / E I, L$ is the beam length, $m$ the mass per unit length, $\omega$ is the circular frequency and $E I$ are respectively the Young modulus and the moment of inertia. Constants $A_{1}-A_{4}$ must be evaluated so as to satisfy the known boundary conditions at the ends of the beam:

$v(0)=0 ; \quad E I \frac{\partial^{2} v(L)}{\partial x^{2}}=K \frac{\partial v(L)}{\partial x} ; \quad v(L)=0 ; \quad E I \frac{\partial^{2} v(L)}{\partial x^{2}}=0 ;$ 
Taking this action, three of the four constants can be expressed in terms of the fourth and the frequency equation can be obtained from which the frequency parameter $\alpha$ is determined [24]. The frequency equation takes the form:

$2 \alpha^{2} \sin (\alpha L) \sinh (\alpha L)-\alpha \frac{K}{E I}\left[\frac{\cosh (\alpha L)}{\sinh (\alpha L)} \sin (\alpha L)-\cos (\alpha L)-\cosh (\alpha L)-\frac{\cosh (\alpha L)}{\sinh (\alpha L)}\right]=0$

and mode shapes are thus obtained according to the following equation:

$$
\begin{aligned}
v_{\alpha_{k}}= & \cos \left(\bar{\alpha}_{k} x\right)+\frac{\cos \left(\bar{\alpha}_{k} L\right)+\cosh \left(\bar{\alpha}_{k} L\right)+\cosh \left(\bar{\alpha}_{k} L\right) / \sinh \left(\bar{\alpha}_{k} L\right)}{\sin \left(\bar{\alpha}_{k} L\right)} \cdot \sin \left(\bar{\alpha}_{k} x\right)- \\
& -\cosh \left(\bar{\alpha}_{k} x\right)+\frac{\cosh \left(\bar{\alpha}_{k} L\right)}{\sinh \left(\bar{\alpha}_{k} L\right)} \sinh \left(\bar{\alpha}_{k} x\right)
\end{aligned}
$$

Without loss of generality and for the sake of simplicity, the value $K / E I$ is taken equal to 1 . In Eq. 24, $\bar{\alpha}_{k}$ is the $k$-th solution of Eq. 23. The solution of Eq. 24 provides for the reference mode shapes; they are reported in Figure 2. It is remarkable the asymmetric mode profiles due to the spring at the right end.

The results of the placement procedure are those already given in Figure 1. The accuracy of the results is evaluated considering the actual mode shapes, that imply a distortion with respect to the simply-supported beam modes due to the rotational spring. In Table 3 and 4 , the values of assessment criteria are reported and the results with the spring at the left or at the right end are given, considering both 4 and 5 mode shapes. With the supposed model form uncertainty, Case $\mathrm{C}$ appears more efficient than the others for all assessment criteria and also for the summary index IR; only when 4 modes are considered, Case A gives a value slightly higher than the proposed formulation when the criterion based on the FIM is considered.

Table 3. Example no. 1 with the spring at one end. Assessment criteria values - 4 modes, 7

$$
\text { sensors }
$$

\begin{tabular}{ccccc}
\hline \multicolumn{5}{c}{ spring at the left end } \\
\hline & $\operatorname{det}($ FIM) & RMS & CN & IR \\
\hline A & 196.78 & 0.035 & 1.68 & 0.613 \\
B & 39.06 & 0.039 & 1.48 & 0.372 \\
C & 178.63 & 0.005 & 1.17 & 0.969 \\
\hline
\end{tabular}

\begin{tabular}{ccccc}
\hline \multicolumn{5}{c}{ spring the at right end } \\
\hline & $\operatorname{det}($ FIM) & RMS & CN & IR \\
\hline A & 194.24 & 0.032 & 1.66 & 0.618 \\
B & 58.20 & 0.018 & 1.41 & 0.467 \\
C & 180.21 & 0.005 & 1.16 & 0.976 \\
\hline
\end{tabular}

\begin{tabular}{|c|c|c|c|c|c|c|c|c|c|}
\hline \multicolumn{5}{|c|}{ spring at the left end } & \multicolumn{5}{|c|}{ spring the at right end } \\
\hline & $\operatorname{det}(F I M)$ & RMS & $\mathbf{C N}$ & IR & & $\operatorname{det}(F I M)$ & RMS & $\mathbf{C N}$ & IR \\
\hline $\mathbf{A}$ & 11.40 & 0.181 & 13.33 & 0.047 & $\mathbf{A}$ & 12.89 & 0.204 & 13.03 & 0.047 \\
\hline B & 62.47 & 0.087 & 2.20 & 0.242 & $\mathbf{B}$ & 61.88 & 0.060 & 2.32 & 0.239 \\
\hline $\mathbf{C}$ & 599.36 & 0.005 & 1.24 & 1.000 & $\mathbf{C}$ & 605.91 & 0.005 & 1.23 & 1.000 \\
\hline
\end{tabular}

Table 4. Example no. 1 with the spring at one end. Assessment criteria values - 5 modes, 7 sensors 
It is finally worth to note that the results could be sensitive to the spring position (left end or right end) if the number of sensors changes. Indeed, in the reference solution (i.e. without the model uncertainty), two solutions symmetrically laid-out with respect to the midspan occur and when the optimal sensor placement procedure was applied, one of the two optimal solutions has been found. The choice of one of them is irrelevant when exact mode shapes are considered; however, it could be crucial if the model error is introduced, remarking the importance of investigate the role of model errors in the sensor positioning process.

\subsection{Example 2: spatial frame unsymmetrical in plan}

Usually, the prediction error is considered totally uncorrelated in two different directions. However, the measurements are correlated if coupled torsional-flexural mode shapes occur e.g. in asymmetric spatial structures. To investigate the role of model errors in $3 \mathrm{D}$ structures, the sensor placement procedure is applied to an eight floor spatial frame with unsymmetrical distribution in plan of columns (see Fig. 3). Considering a rigid diaphragm at each floor, the modal matrix is written considering displacements of centroids for all modes of interest. Mode shapes are mass normalized, and, therefore, first modes assume much more relevance.

Floor decks are subdivided in a grid of $5 \times 5$ possible sensor positions. The placement procedure is applied considering 6 or 12 sensors; the results obtained by case $C$ are reported in Figure 3 and they are compared to those obtained by the case A (i.e. the covariance matrices is assumed equal to the identity matrix). In Figure 3 the case of 12 sensors is considered; sensors are indicated with an increasing number depending on the placement order. The first five sensors are placed in same positions in both cases; sensors 1-3 are placed at the top floor, in both directions, allowing to identify the floor rigid motion. In addition, sensors are placed on edges, avoiding inner position to gather floor rotation as well. Subsequent sensors are then positioned at lower levels, allowing to identify also higher modes. In the proposed procedure (see Figure $3 b$ ) sensors are well distributed on the structure; as expected, results of the case A (Figure $4 a$ ) show a concentration of sensors on the top floor, despite the $4^{\text {th }}, 6^{\text {th }}$ and $7^{\text {th }}$ floors that are completely unmonitored.

Similarly, to results shown in the Example 1, the three assessment criteria confirm that the proposed formulation gives better performances especially for a high number of modes.

Parametric uncertainties are then introduced; the sensor placement procedure is performed starting from a set of pseudo-experimental data, simulating variations in distribution of floor masses. Pseudoexperimental data are obtained by multiplying exact values of floor masses by uncorrelated coefficients, extracted from normal probability distributions with unit mean value and coefficient of variation equal to 40 percent. A Monte Carlo simulation is performed considering 100 tests.

It is worth of note that a coefficient of variation equal to $40 \%$ could generate negative mass values. If it happens, the simulation is dismissed and all values of masses are re-extracted. The Monte Carlo approach requires a large number of simulations and results in a high computational effort. However, the computational cost and time for the simple structure here considered is very small and therefore the method is considered suitable for the aim of this case study.

Statistical results obtained from tests are reported in Figure 4. The number of modes and the number of sensors are selected equal to 6. In Figure 4, the x-axis reports the Degree-of-Freedom (DOF) number while the number of occurrence over 100 tests for the selected DOF is reported in the 
y-axis; the DOF are numbered as follows: a value from 1 to 200 indicates a DOF in the $\mathrm{x}$ direction and from 201 to 400 in the y direction; for each floor 25 DOF are considered and they are numbered starting from the first floor to the top one. The results show that there are 3 DOF that are always chosen as optimal sensor location. These sensor locations are of great importance for the success of the identification process. As expected, they are placed at the top floor and they allow to identify the top floor rigid motion. In Figure 4 they are indicated with the DOF 196, 376 and 380. Nevertheless, higher dispersion of results is instead found for the next sensors. The higher dispersion is due to the random variation of input data: the changing of the input modal parameters modifies the position of the global minimum of objective function; therefore, the sensor positions at lower floors vary because they are highly sensitive to changes of second bending modes. If the proposed correlation length is used (case C, see Figure 4b), the variation of results at lower floors is less sensitive to parameter changes, identifying some preferred position. On the other hand, if case A is considered, an almost uniform distribution of sensors is found and the choice of the set of measurement positions is a very difficult task, even more so if adjacent points have to be avoided since they usually do not provide useful information [1].
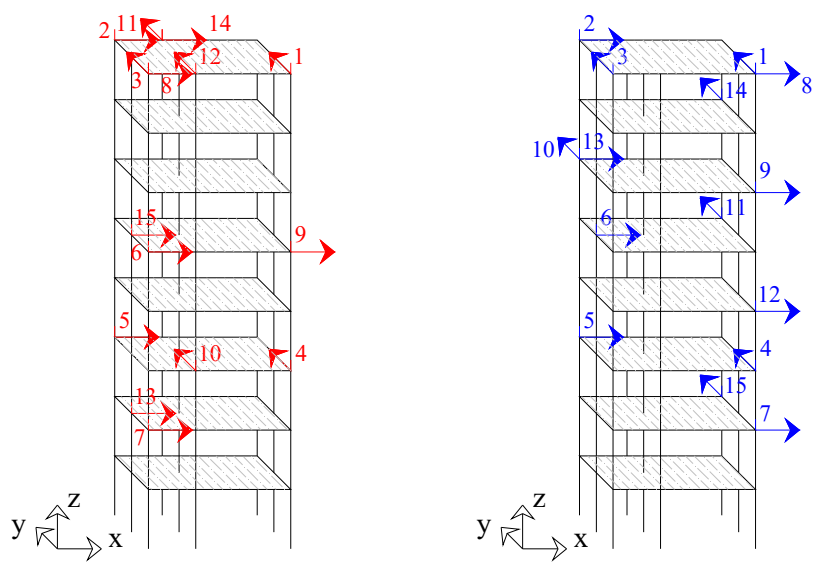

(a)

(b)

Figure 3 Example no. 2: optimal sensor position on the spatial frame for $(a)$ case A and $(b)$ case C.

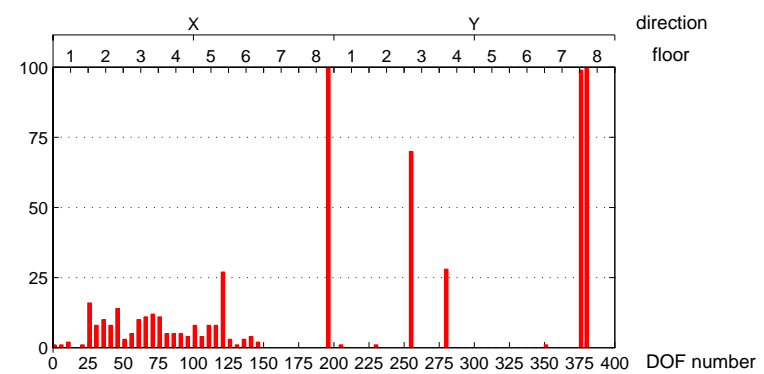

Case A

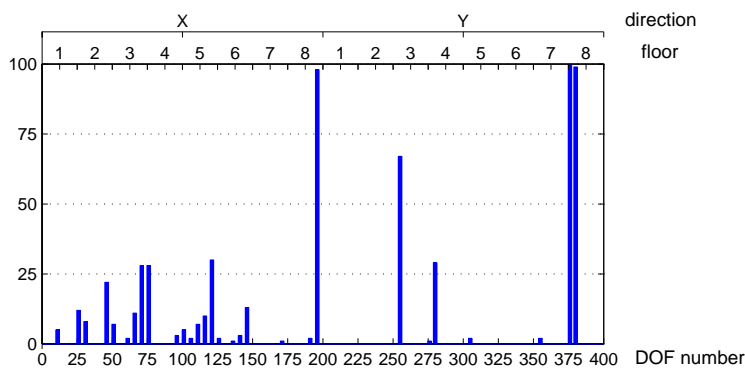

Case C

Figure 4. Example no. 2: Distribution of sensors for the case A and C- 6 modes and 6 sensors 
Table 5. Example no. 2 Assessment criteria values -6 and 9 modes, 12 sensors

\begin{tabular}{cccc}
\hline \multicolumn{4}{c}{ 6 modes } \\
\hline & $\operatorname{det}($ FIM) & RMS & CN \\
\hline $\mathrm{A}$ & 1.610 & 0.175 & 65.63 \\
$\mathrm{C}$ & 1.084 & 0.174 & 67.93 \\
\hline
\end{tabular}

\begin{tabular}{cccc}
\hline \multicolumn{4}{c}{9 modes } \\
\hline & $\operatorname{det}($ FIM) & RMS & CN \\
\hline $\mathrm{A}$ & $-1.12 \mathrm{E}-24$ & 0.29 & $7.9 \mathrm{E}+09$ \\
$\mathrm{C}$ & $2.81 \mathrm{E}-06$ & 0.17 & 236.31 \\
\hline
\end{tabular}

\section{Sensor placement on the Correggio Footbridge.}

Optimal sensor configuration is finally designed for a real footbridge located in Correggio (Italy). The Correggio steel footbridge (Figure 5) was built in 2011; it is $168.0 \mathrm{~m}$ long and it is composed of 5 simple-supported spans, linked at lower-floor level. The central span is $40.0 \mathrm{~m}$ long and the four lateral spans are $32.0 \mathrm{~m}$ long each one. The footbridge is supported by 4 piles; the central and lateral piles are about $5.20 \mathrm{~m}$ and $2.80 \mathrm{~m}$ high, respectively. The structure has a box cross-section composed of truss girders; the deck is $3.00 \mathrm{~m}$ wide and consists of a wooden plank resting on a corrugated sheet. L-shaped profiles are used for the lower lateral bracing system. Piles and trusses are made up by rectangular hollow profiles. The whole structure is slender, lightweight and it suffers notable vibrations under the pedestrian action. The design and implementation of a monitoring system is thus necessary to investigate the interaction between pedestrians and the footbridge [35]. For this purpose, a dynamic identification [36] and a model updating [32] procedures are needed, and so, the goal of the sensor location design is the modal identification. A finite element model is built to find numerical mode shapes. Truss and beam elements are used for girders and piles; constraints are applied at the base of piles and at the lateral span ends.

The design of sensor position is performed considering vertical and mixed lateral-torsional mode shapes. The firsts 10 natural frequencies are reported in Table 6 and the firsts 6 mode shapes are illustrated in Figure 6. The correlation function proposed in this paper is employed; mass-normalized mode shapes are used in eq. 13 to calculate the covariance matrix elements. In order to emphasize the importance of a spatial correlated prediction errors in the identification of the optimal sensor location, three modal cases (called 1, 2 and 3, respectively) are considered. In modal case 1, only vertical modes are considered; in modal case 2, modes involving mainly in the horizontal direction (torsional or lateral modes) are selected; finally, in modal case 3 all firsts 10 modes are taken into account.
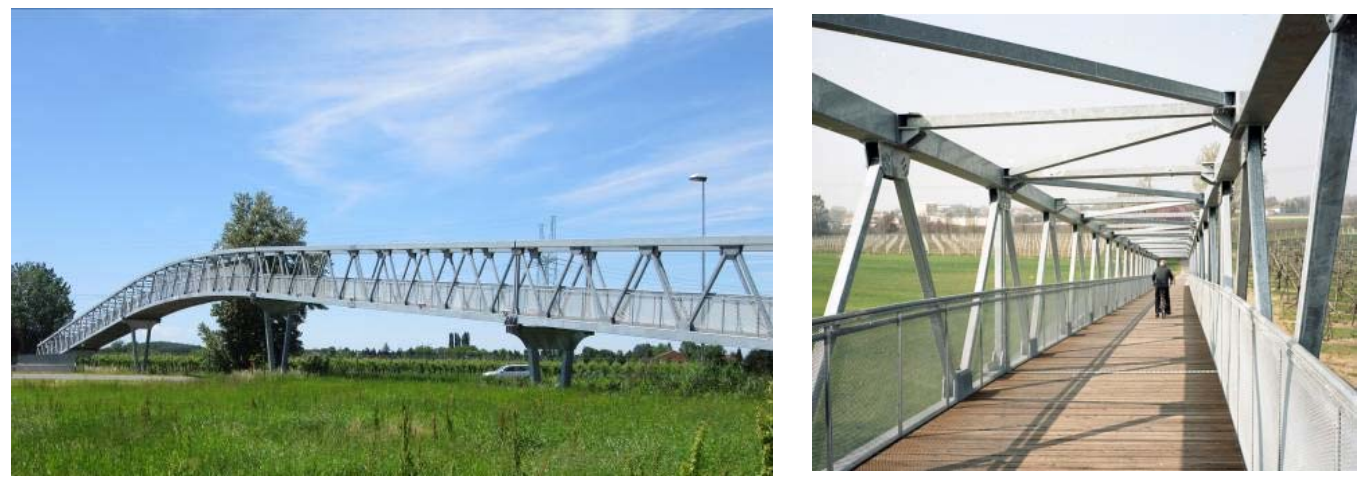

Figure 5. The Correggio Footbridge 
Table 6. Correggio footbridge: Frequency and mode shapes

\begin{tabular}{ccc}
\hline Mode no. & Frequency $[\mathrm{Hz}]$ & Mode shape \\
\hline 1 & 1.676 & $1^{\text {st }}$ lateral, global \\
2 & 2.702 & $2^{\text {nd }}$ lateral, global \\
3 & 3.103 & $3^{\text {rd }}$ lateral, global \\
4 & 4.293 & $1^{\text {st }}$ vertical, global \\
5 & 5.236 & $1^{\text {st }}$ torsional, central span \\
6 & 5.520 & $2^{\text {nd }}$ vertical, lateral spans \\
7 & 6.476 & $3^{\text {rd }}$ vertical, lateral spans \\
8 & 6.521 & $2^{\text {nd }}$ torsional, global \\
9 & 8.354 & $3^{\text {rd }}$ torsional, global \\
10 & 11.063 & $4^{\text {th }}$ vertical, central span \\
\hline
\end{tabular}

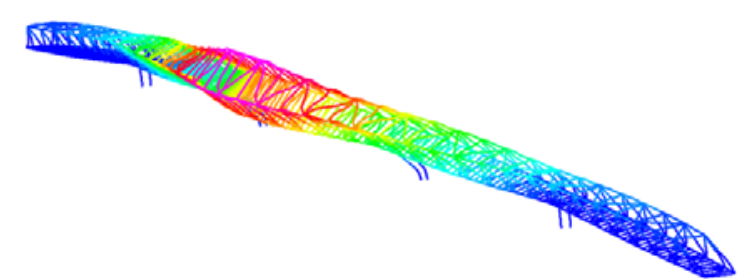

Mode no. $1-1^{\text {st }}$ lateral, global $-1.676 \mathrm{~Hz}$

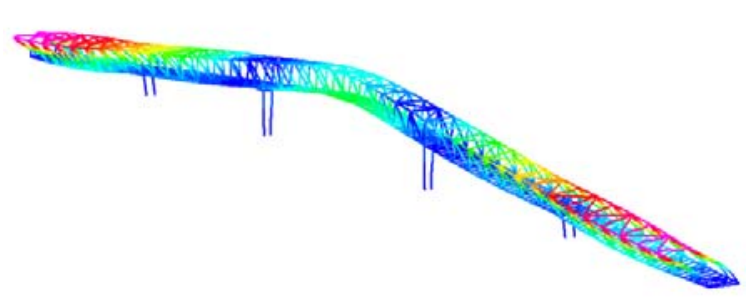

Mode no. 3 - $3^{\text {rd }}$ lateral, global $-3.103 \mathrm{~Hz}$

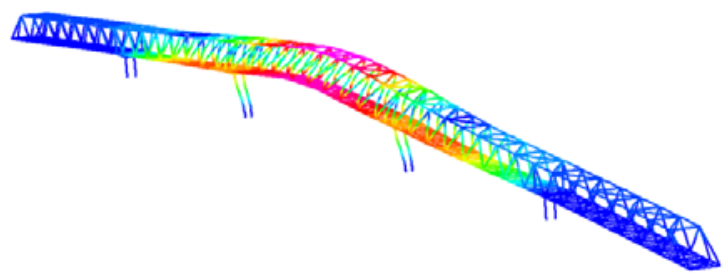

Mode no. 5 - $1^{\text {st }}$ torsion, central span - 5.236

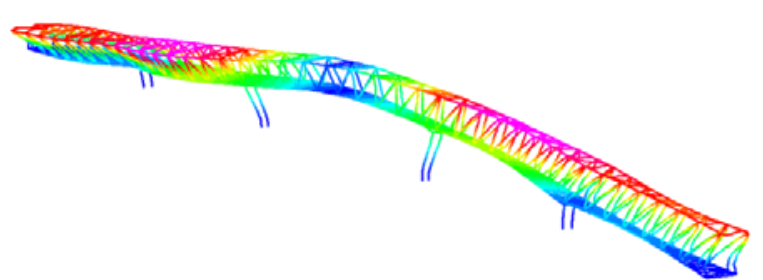

Mode no. 2 - $2^{\text {nd }}$ lateral, global $-2.702 \mathrm{~Hz}$

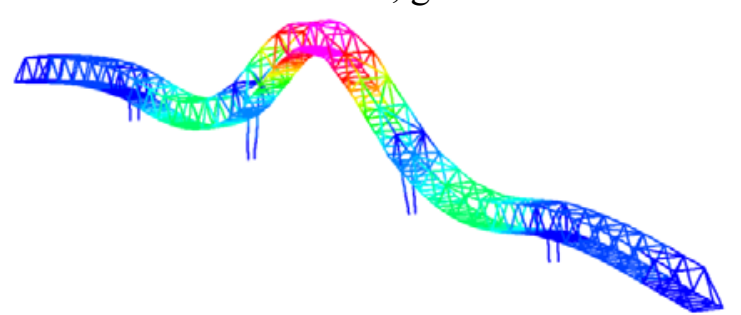

Mode no. $4-1^{\text {st }}$ vertical, global $-4.293 \mathrm{~Hz}$

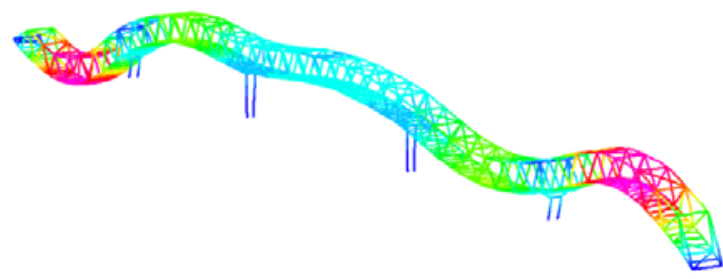

Mode no. 6 - $2^{\text {nd }}$ vertical, lateral spans - 5.52

Figure 6 Correggio footbridge: first 6 mode shapes

The methodology is applied using the proposed correlation function and results are shown in Figures 7-9. For the sake of simplicity, spans of the bridge are denoted with roman numbers; Y and $\mathrm{Z}$ indicates vertical and lateral direction, respectively. The increasing number indicates the placement order obtained by the FSSP procedure. 
In modal case 1, sensors are obviously placed all in the vertical direction, on both side of the footbridge, well distributed over all spans and approximately symmetrically laid out with respect to the central span. The central span exhibits a higher number of sensors, due to the form of the selected mode shapes. In modal case 2, sensors are placed on the upper and lower chord of the box truss girder, in order to better identify the torsion involved in modes no. 5, 8 and 9. With the same purpose, two sensors are also placed in the vertical direction at the midspan (sensors Y19 and Y20). At last, the modal case 3 shows sensors in both $\mathrm{Y}$ and $\mathrm{Z}$ direction and approximately symmetric placed respect to the midspan. In Figure 10 the MAC matrix obtained for the third modal case is reported. Diagonal values are obviously equal to 1 ; on the contrary, off-diagonal terms are very small with the exception of few values, which confirm the good disposition of sensors; these non-negligible values are associated to a correlation between first lateral mode and first torsional mode and between second lateral and third torsional modes.

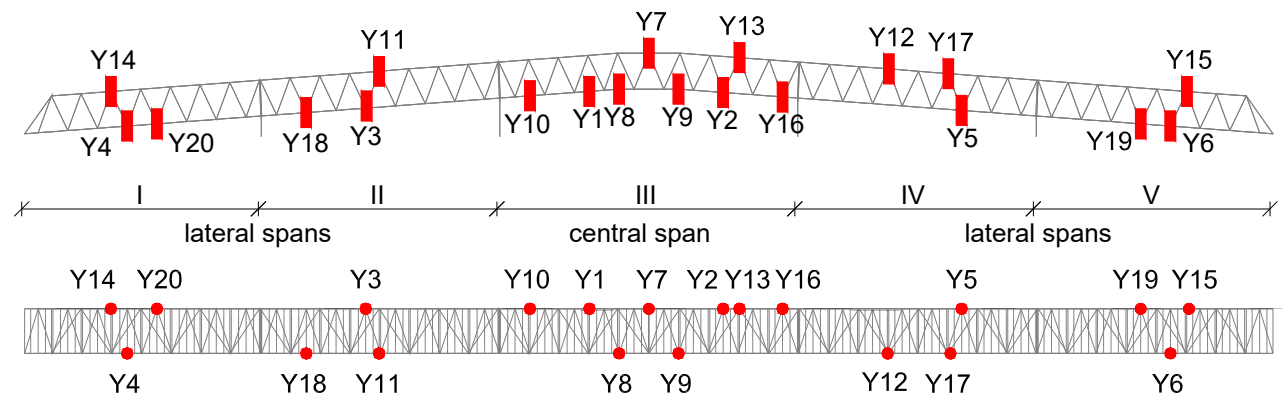

Figure 7 Modal case 1: Optimal sensor position selecting only vertical modes

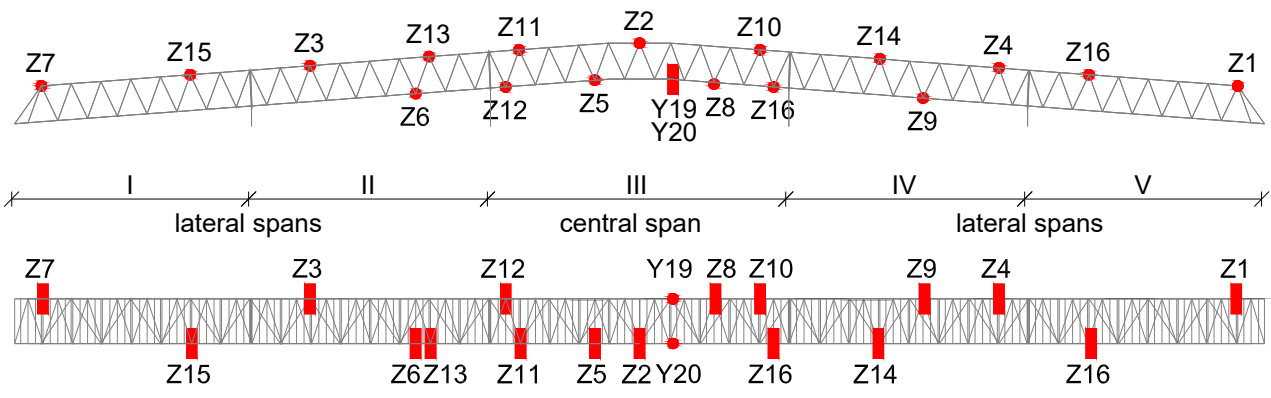

Figure 8 Modal case 2: Optimal sensor position selecting only lateral and torsional modes

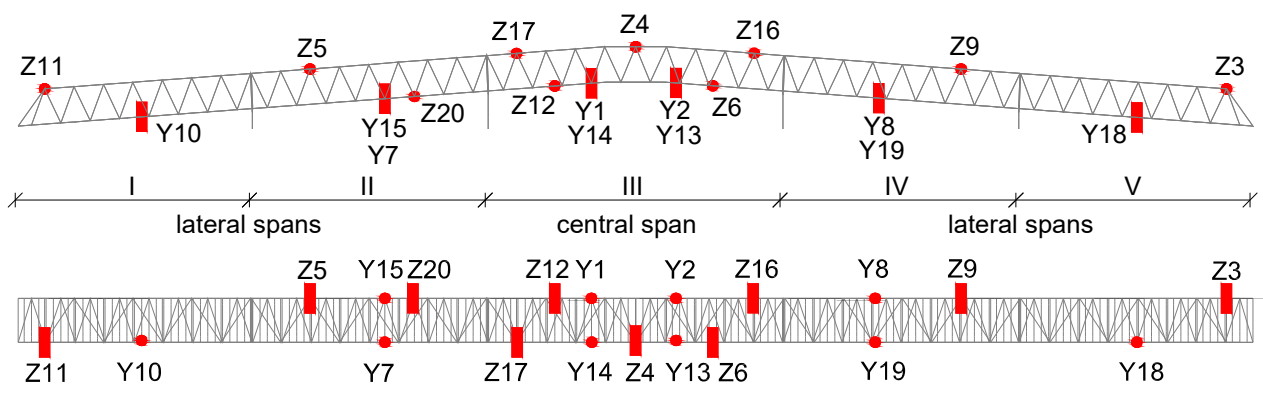

Figure 9 Modal Case 3: Optimal sensor position selecting the first ten modes. 
Finally, the influence of model form uncertainties is investigated. The upper chords of spans are connected together by means of a thin steel plates and they are bolted in slotted holes. To model the connection, beam elements with stiffness klink are introduced in the Finite Element model. Actually, a stiffness $k_{\text {link }}$ change modifies mode shapes, especially those mainly involved in the lateral direction; the effective stiffness of the connection is unknown, and so, the value used in the analysis could be considered affected by uncertainty. For this reason, the influence in sensor positions is investigated considering different values of klink by changing its elastic modulus in the range $E_{\text {link }}=\left[10^{7} \mathrm{~Pa}, 10^{11}\right.$ Pa]. Mode shapes number 1, 2 and 4 obtained with $E_{\text {link }}=10^{7} \mathrm{~Pa}$ and $E_{\text {link }}=10^{11} \mathrm{~Pa}$ are reported in Figure 11.

The results are reported in Figure 12 for the three modal cases described previously, where each dot indicates the position obtained by the sensor placement process for a given value of the elastic modulus $E_{\text {link. }}$. Three modes are considered for modal cases 1 and 2, while 10 modes are selected in modal case 3 . An elastic modulus $E_{\text {link }}$ change could significantly modify mode shapes and their order. For this reason, modes with the highest participation masses in the vertical or horizontal direction are selected as the input for the sensor placement process. The firsts 10 sensor positions are reported in Figure 12 a-c for modal cases 1-3, respectively. It is shown that 9 sensors remain almost in the same positions if vertical modes are considered (Figure 12a); sensors are placed in all spans, close to the each midspan. Moreover, three sensors are placed in the span III, to identify better the mode shapes no. 6 for which the midspan is a nodal point (that is point of no displacement for the mode shape). As expected, greater variability of the results is instead obtained when horizontal mode shapes are considered (Figure 12b). The first two sensors are always placed at the footbridge ends, in order to identify the second and third lateral modes; to identify the first mode shape, the third sensor is placed in the span III, sometimes paired with the forth sensor. Other sensors are instead spread in all spans. For small values of $E_{\text {link }}$ the first 2 mode shapes have similar displacements in all DoF of spans II, III and IV (see Figure 11b,d). Therefore, a small variation of Elink causes large variation of sensor positions. More stable results are obtained for modal case 3, due to the increasing number of selected modes. With exception to the lowest values of $E_{\text {link, }}$, sensors are placed at each midspan in the vertical directions, and at the footbridge ends in the horizontal direction.

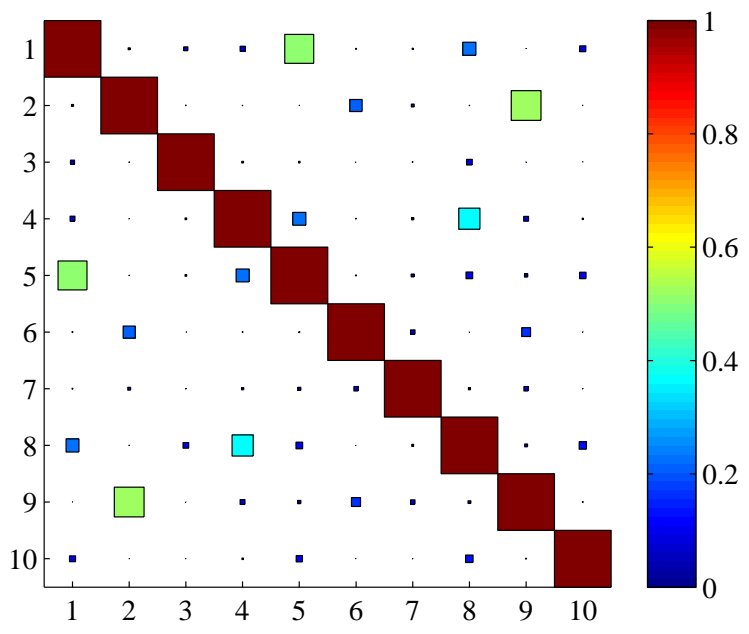

Figure 10 Modal Assurance Criterion (MAC) of the first ten modes 


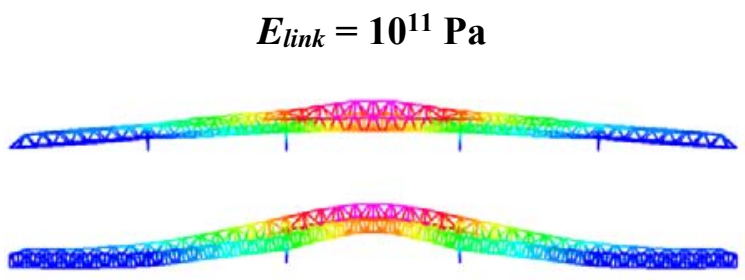

(a) mode no. 1

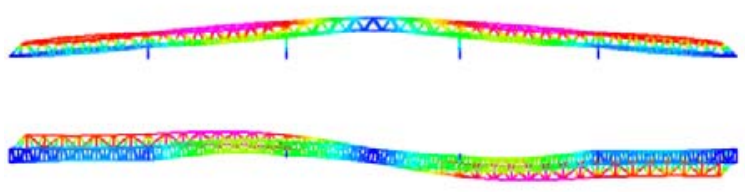

(c) mode no. 2

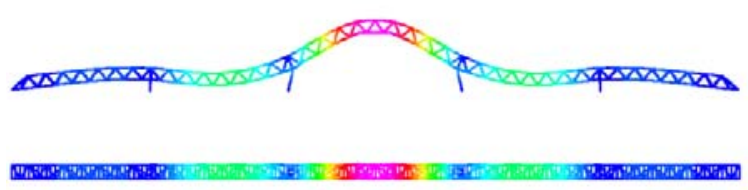

(e) mode no. 4

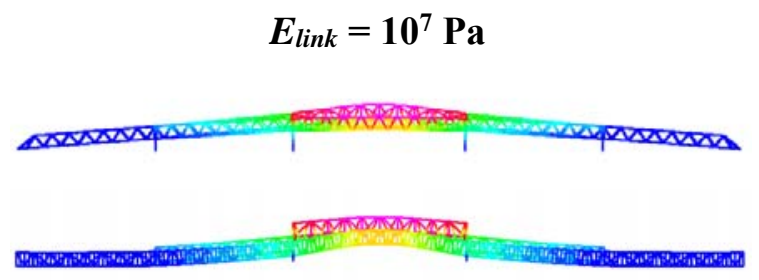

(b) mode no. 1

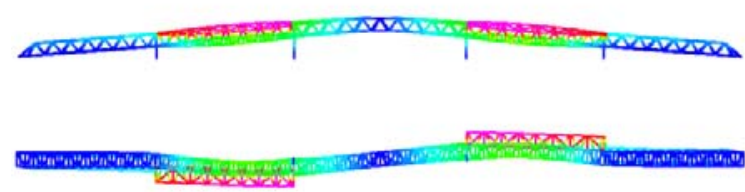

(d) mode no. 2

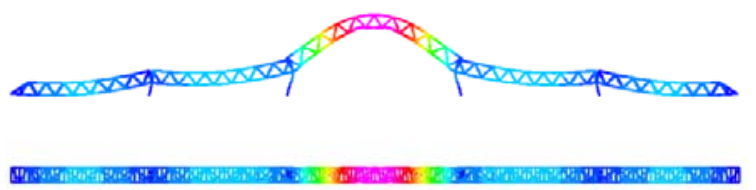

(f) mode no. 4

Figure 11 Correggio footbridge: plan and elevation of mode shapes no. 1, 2 and 5 obtained with $(a, c, e) E_{\text {link }}=10^{11} \mathrm{~Pa}$ and $(b, d, f) E_{\text {link }}=10^{7}$

\section{Conclusions}

The paper investigates the role of the covariance matrix and the correlation function in optimal or near optimal sensor placement for structural health monitoring and modal testing. The optimal set of measurement locations is obtained by the Information Entropy theory and the solution of the optimization problem is obtained maximizing the determinant of the Fisher Information Matrix. The problem of computing the Fisher information matrix determinant has been solved performing the Singular Value Decomposition technique and the Cholesky factorization when the number of sensors is less than the number of contributing modes of interest. The results of placement process considerably depend on the so called covariance matrix of prediction error as well as on the definition of the correlation function. Firstly, it is assumed a constant and an exponential correlation function which depends on the distance between sensors; then it is presented a new proposal which depends on both the distance and the modal vectors.

Two simple numerical case studies are presented to investigate the role played by the covariance matrix of the prediction error and how uncertainties in the structural model could modify the results of the sensor placement. The first case study refers to a simply supported beam. The results obtained by the investigated methods are similar when the problem is fully determined; however, our proposal better estimates also higher modes when the number of sensors is greater than the number of modes. The model form uncertainty is also considered forcing an alteration in a constraint condition. The proposal appears more efficient with higher values of the summary index IR. In the second case, the proposed correlation function is applied with reference to an eight floors asymmetric-plan building; 


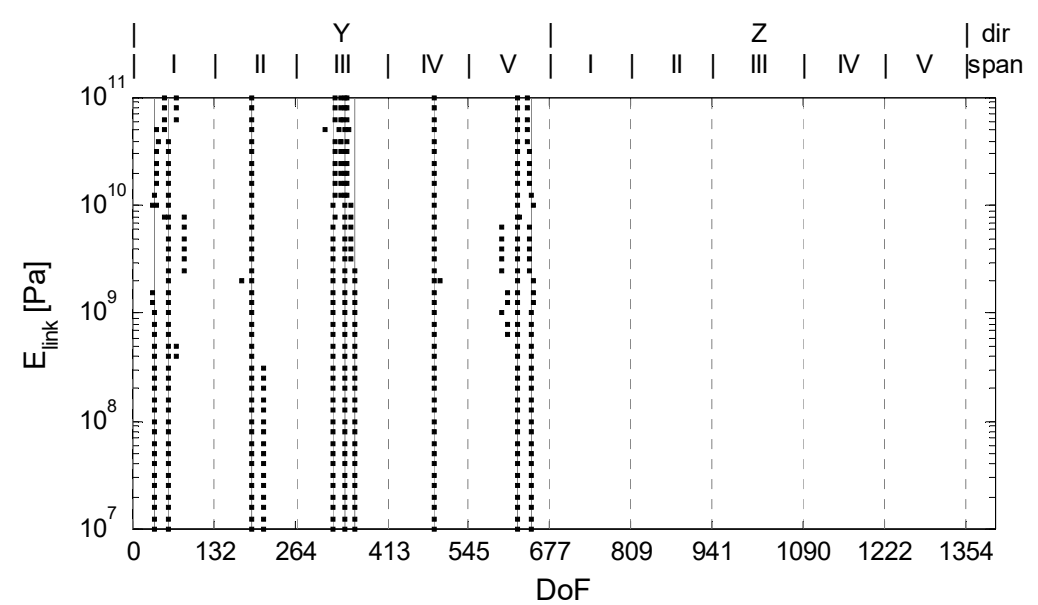

(a)

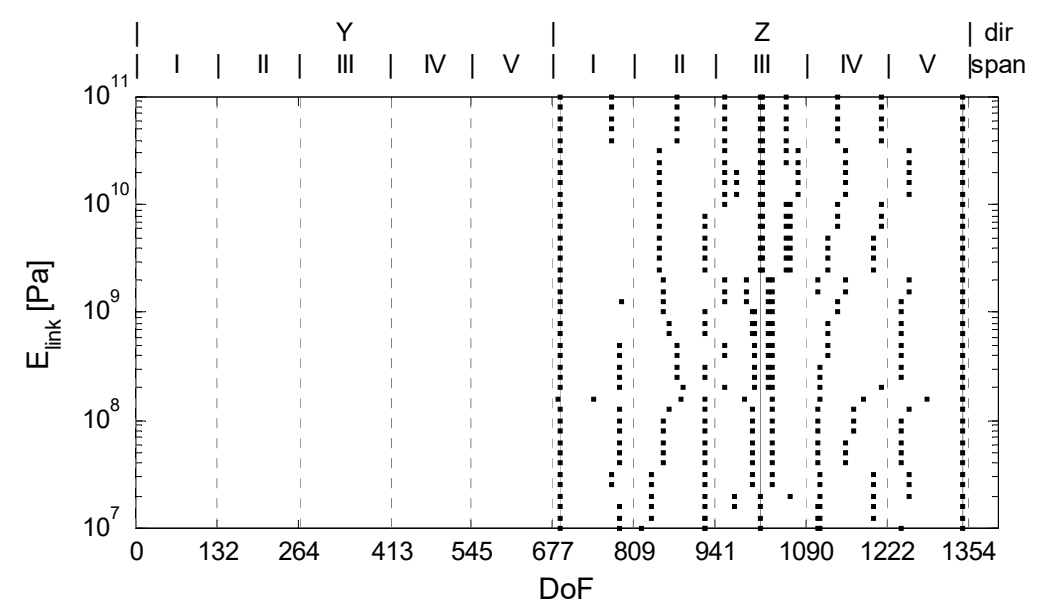

(b)

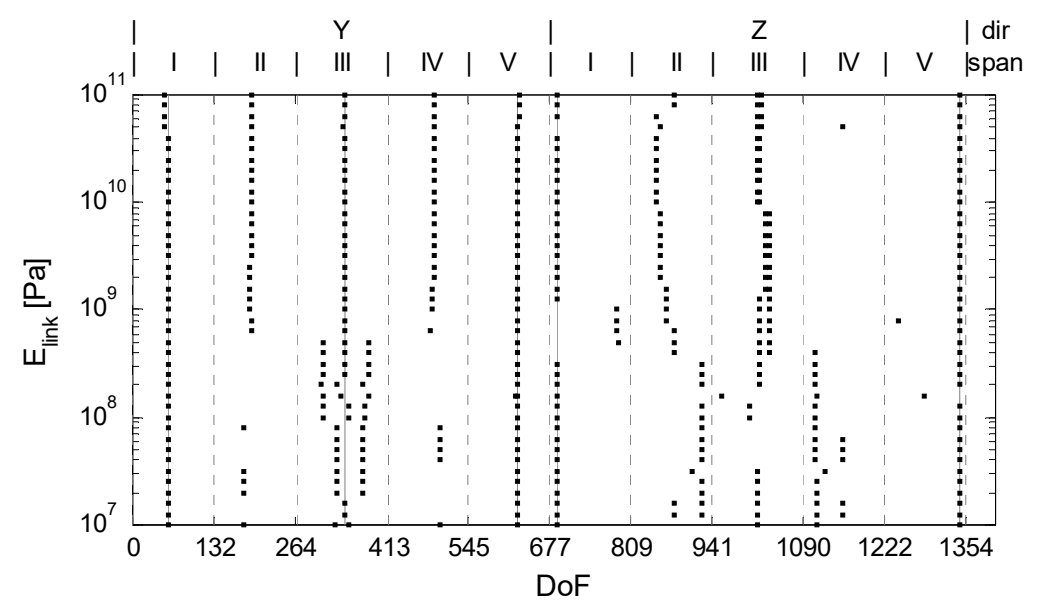

(c)

Figure 12 Correggio footbridge: results of sensor placement in (a) modal case 1- vertical modes, (b) modal case 2 - horizontal modes, and (c) modal case 3 - all modes 
it is shown that the proposed correlation function easily allows to take into account the spatial correlation in 3D structures. Finally, optimal sensor locations are designed for the footbridge in Correggio (Italy). Vertical and mixed lateral-torsional mode shapes are considered. The correlation function proposed in this paper is applied and the influence of model form uncertainties is investigated by changing the elastic modulus of an element linking the upper chords of the footbridge. The results of the sensors placement show that vertical sensors remain almost in the same positions even if the number of sensors is fairly greater than the number of selected modes. Greater variability of the results is instead obtained for horizontal sensors; after placing the three sensors needed to identify the 3 selected horizontal mode shapes, other sensor positions show large fluctuations, caused by similar component values of mode shapes for the DoFs of the same spans, especially when the stiffness of links is small.

\section{Acknowledgements}

The research was developed in the framework of the Reluis 2015 research project - Task RS4: "Seismic observatory of structures and structural health monitoring". The financial supports of the Italian Department of Civil Protection is gratefully acknowledged.

\section{References}

[1] R. Castro-Triguero, S. Murugan, R. Gallego, M.I. Friswell, Robustness of optimal sensor placement under parametric uncertainty, Mechanical Systems and Signal Processing, 41 (2013) 268-287.

[2] C. Papadimitriou, Optimal sensor placement methodology for parametric identification of structural systems, Journal of Sound and Vibration, 278 (2004) 923-947.

[3] C. Papadimitriou, G. Lombaert, The effect of prediction error correlation on optimal sensor placement in structural dynamics, Mechanical Systems and Signal Processing, 28 (2012) 105127.

[4] V. Gupta, M. Sharma, N. Thakur, Optimization Criteria for Optimal Placement of Piezoelectric Sensors and Actuators on a Smart Structure: A Technical Review, Journal of Intelligent Material Systems and Structures, 21 (2010) 1227-1243.

[5] R.J. Allemang, D.L. Brown, A correlation coefficient for modal vector analysis, in: Proceedings of the1st International Modal Analysis Conference IMAC, 1982, pp. 110-116.

[6] T.G. Carne, C.R. Dohrmann, A Modal Test Design Strategy for Model Correlation, in: IMAC XIII - 13th International Modal Analysis Conference, 1995

[7] J.E.T. Penny, M.I. Friswell, S.D. Garvey, Automatic choice of measurement locations for dynamic testing, AIAA Journal, 32 (1994) 407-414.

[8] W. Liu, W.-c. Gao, Y. Sun, M.-j. Xu, Optimal sensor placement for spatial lattice structure based on genetic algorithms, Journal of Sound and Vibration, 317 (2008) 175-189.

[9] D. Borissova, I. Mustakerov, L. Doukovska, Predictive Maintenance Sensors Placement by Combinatorial Optimization, International Journal of Electronics and Telecommunications, 58 (2012) 153-158.

[10] M. Meo, G. Zumpano, On the optimal sensor placement techniques for a bridge structure, Engineering Structures, 27 (2005) 1488-1497

[11] D.C. Kammer, R.D. Brillhart, Optimal sensor placement for modal identification using systemrealization methods, AIAA journal, (1994). 
[12] D.C. Kammer, Sensor Placement for On-Orbit Modal Identification and Correlation of Large Space Structures, in: American Control Conference, 1990, 1990, pp. 2984-2990.

[13] D.C. Kammer, L. Yao, Enhancement of On-Orbit Modal Identification of Large Space Structures Through Sensor Placement, Journal of Sound and Vibration, 171 (1994) 119-139.

[14] D.-S. Li, H.-N. Li, C.-P. Fritzen, A note on fast computation of effective independence through QR downdating for sensor placement, Mechanical Systems and Signal Processing, 23 (2009) $1160-1168$.

[15] D.C. Kammer, J.A. Peck, Mass-weighting methods for sensor placement using sensor set expansion techniques, Mechanical Systems and Signal Processing, 22 (2008) 1515-1525.

[16] A.R.M. Rao, G. Anandakumar, Optimal sensor placement techniques for system identification and health monitoring of civil structures, Smart Structure and Systems, 4 (2008) 465 - 492.

[17] M. Reynier, H. Abou-Kandil, Sensors location for updating problems, Mechanical Systems and Signal Processing, 13 (1999) 297-314.

[18] E. Heredia-Zavoni, L. Esteva, Optimal instrumentation of uncertain structural systems subject to earthquake ground motions, Earthquake Engineering \& Structural Dynamics, 27 (1998) 343362.

[19] C. Stephan, Sensor placement for modal identification, Mechanical Systems and Signal Processing, 27 (2012) 461-470.

[20] P.C. Shah, F.E. Udwadia, A methodology for optimal sensor locations for identification of dynamic systems, ASME, Transactions, Journal of Applied Mechanics, 45 (1978) 188-196.

[21] T. Hanis, M. Hromcik, Optimal sensors placement and spillover suppression, Mechanical Systems and Signal Processing, 28 (2012) 367-378.

[22] C. Papadimitriou, J.L. Beck, S.-K. Au, Entropy-Based Optimal Sensor Location for Structural Model Updating, Journal of Vibration and Control, 6 (2000) 781-800.

[23] G.-H. Feng, Y.-L. Pan, Establishing a cost-effective sensing system and signal processing method to diagnose preload levels of ball screws, Mechanical Systems and Signal Processing, 28 (2012) 78-88.

[24] R.W. Clough, J. Penzien, Dynamics of structures, Third Edition ed., Computers \& Structures, Inc., 2003.

[25] S.W. Doebling, Measurement of Structural Flexibility Matrices for Experiments with Incomplete Reciprocity, University of Colorado, 1995.

[26] V.V. Fedorov, P. Hackl, Optimal experimental design: spatial sampling, Bulletin of the Calcutta Statistical Association, 44 (1994) 57-82.

[27] R.F. Guratzsch, Sensor placement optimization under uncertainty for Structural Health Monitoring systems of hot aerospace structures, in, Vanderbilt University, 2007.

[28] P.H. Kirkegaard, R. Brincker, On the optimal location of sensors for parametric identification of linear structural systems, Mechanical Systems and Signal Processing, 8 (1994) 639-647.

[29] C.E. Shannon, A mathematical theory of communication, The Bell System Technical Journal, 27 (1948) 379-423.

[30] R. Guidorzi, R. Diversi, L. Vincenzi, C. Mazzotti, V. Simioli, Structural monitoring of a tower by means of MEMS-based sensing and enhanced autoregressive models, European Journal of Control, 20 (2014) 4-13.

[31] L. Vincenzi, G. De Roeck, M. Savoia, Comparison between coupled local minimizers method and differential evolution algorithm in dynamic damage detection problems, Advances in

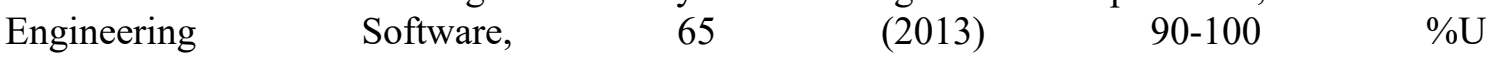
http://www.sciencedirect.com/science/article/pii/S0965997813000938.

[32] L. Vincenzi, M. Savoia, Coupling Response Surface and Differential Evolution for Parameter Identification Problems, Computer-Aided Civil and Infrastructure Engineering, 30 (2015) 376393. 
[33] H. Bedrossian, S.F. Masri, Optimal placement of sensors \& shakers for modal identification, in: Computational Stochastic Mechanics, P.D. Spanos \& G. Deodatis, Corfu, Greece, 2003, pp. 5357

[34] D.J. Ewins, Modal testing: theory and practice, John Wiley \& Sons, New York, 2000.

[35] E. Bassoli, P. Gambarelli, L. Simonini, L. Vincenzi, Dynamic analyses of a curved cable-stayed footbridge under human induced vibrations: Numerical models and experimental tests, in: COMPDYN 2015 - 5th ECCOMAS Thematic Conference on Computational Methods in Structural Dynamics and Earthquake Engineering, 2015, pp. 2495-2511.

[36] M. Savoia, L. Vincenzi, E. Bassoli, P. Gambarelli, R. Betti, R. Testa, Identification of the Manhattan bridge dynamic properties for fatigue assessment, in: Safety, Reliability, Risk and Life-Cycle Performance of Structures and Infrastructures, CRC Press, 2014, pp. 4667-4674 